\title{
Hipparcos data supporting the IB school curriculum
}

\author{
K. S. O'Flaherty ${ }^{1}$, A. Brumfitt ${ }^{2}$ and C. Lawton ${ }^{3}$ \\ ${ }^{1}$ Research \& Scientific Support Dept., Directorate of the Scientific Programme, \\ European Space Agency \\ email: koflaher@rssd.esa.int \\ ${ }^{2}$ Directorate Research and Education Support, Space Qualified Ltd., Australia \\ email: anne.brumfitt@spacequalified.org \\ ${ }^{3}$ Scientific Projects Dept., Directorate of the Scientific Programme, European Space Agency \\ email: Christopher.Lawton@esa.int
}

\begin{abstract}
The Hipparcos and Tycho catalogues are ideal data sources for classwork since they provide real data illustrating fundamental astronomical concepts in a simple and easy to manipulate format. In this poster we describe how some of this data is used as support material within the International Baccalaureate (IB) science curriculum. The preparation and deployment of a series of Teacher Notes, specifically constructed to support the IB but applicable to other curricula, is described. Specific attention is given to the Teacher Notes on Stellar Distances which employs data from the Hipparcos and Tycho catalogues.
\end{abstract}

Keywords. astrometry, sociology of astronomy, education, catalogs

\section{Introduction}

Europe is facing a crisis in science education with a marked decline in the numbers of students opting to study science, particularly physical sciences (OECD, 2006). The resulting deficit in the number of highly qualified science and technology professionals will have important consequences for Europe's prospects as a knowledge-based society.

A key factor governing the decline in the number of students following science courses arises from the way science is, or has been, taught (Eurobarometer, 2005). Within the traditional classroom-based teaching approach methods supporting Inquiry-Based Science Education (IBSE), in which observation and experimentation play a key role, are now acknowledged as one way of rekindling the interest of a new generation of students. Teachers also need to be inspired and supported in their delivery of the science curriculum since they are the cornerstone of any renewal of science education.

The Teacher Notes project (ESA, 2005) was initiated, after consultation with the International Baccalaureate Organisation, with the aim of creating support material for teachers of the IB Astrophysics option using real data from ESA's science missions, in particular from the Hipparcos mission.

\section{The International Baccalaureate science curriculum}

The International Baccalaureate school system (http://www.ibo.org) offers a programme of international education, covering the age range 3 to 19 . The programmes are taught in English, French, Spanish or Chinese in 2,125 IB schools in 125 countries.

For the Teacher Notes project we focussed on the Diploma Programme for students aged 16 to 19. This is a demanding two-year curriculum leading to final examinations 
and a qualification that is recognised by leading universities around the world. Science subjects form part of the mandatory curriculum of this programme.

\section{Teacher notes - support material for the IB astrophysics option}

\subsection{Structure of the teacher notes}

The Teacher Notes closely follow the structure of the IB Astrophysics option. Each topic is introduced and clearly explained using familiar examples, whether from day-to-day life or from other areas of the programme curriculum. Where appropriate, tables of values are given for familiar or standard objects. Clear and simple diagrams are used to illustrate concepts and ideas. Worked examples are provided to familiarise the teacher with using the ESA data, and extended data sets are made available for use in the classroom and for further experimentation by the students. The entire package provides a teacher with the basic information and tools needed to teach the topic. The notes are written in a clear and concise manner, and careful attention has been paid to the language used.

\subsection{Content of the teacher notes}

Within the IB Diploma Programme 15 teaching hours are assigned for the four topics delivered at Standard Level: Introduction to the Universe, Stellar radiation and stellar types, Stellar distances, and Cosmology. An additional 7 hours are assigned for the two extra topics which are taught at Higher Level: Stellar processes and stellar evolution, and Galaxies and the expanding universe.

\subsection{Hipparcos data: use and access}

The Hipparcos and Tycho Catalogues (ESA, 1997) are the primary data product of the European Space Agency's Hipparcos mission. They contain fundamental astronomical parameters such as the positions, proper motions, parallaxes, magnitude, variability, multiplicity, etc., for a large number of stars. The data are of immediate application to the astrophysics option of the IB, in particular to the section dealing with stellar distances. The fact that the data are easy to manipulate is an important consideration for the end users. Available as simple ASCII tables, and requiring no special software programs to extract the fields that are needed, makes them appealing to teachers and students alike. The topic of stellar distances is notable for the emphasis on problem solving. Students must (a) determine distances using parallax, (b) solve problems involving ratios of apparent brightness and of apparent magnitudes, (c) solve problems involving distances, apparent brightness and luminosity, and (d) determine distances to Cepheids using the Period-Luminosity relationship. Extracts of Hipparcos data are provided as data sources for these problems allowing teachers to illustrate the concepts, and students to explore them.

\section{Acknowledgements}

We acknowledge the key contribution to this project of Jo Turner, science writer.

\section{References}

ESA, 1997, The Hipparcos and Tycho Catalogues, ESA SP-1200

ESA, 2005, Teacher Notes, available online at http://sci.esa.int/teachernotes

Eurobarometer, 2005, Europeans, Science \& Technology, Special Eurobarometer 224

OECD, 2006, Evolution of Student Interest in Science and Technology Studies - Policy Report 